\title{
Involvement of circulating endothelial progenitor cells in carotid plaque growth and vulnerability
}

\author{
Daina Kashiwazaki, MD, ${ }^{1}$ Naoki Akioka, MD,1 Naoya Kuwayama, MD, PhD, ${ }^{1}$ Tomohide Hayashi, MD,1 \\ Kyo Noguchi, MD, PhD, ${ }^{2}$ Kortaro Tanaka, MD, PhD, ${ }^{3}$ and Satoshi Kuroda, MD, PhD ${ }^{1}$
}

\begin{abstract}
Departments of ${ }^{1}$ Neurosurgery and ${ }^{2}$ Radiology, Graduate School of Medicine and Pharmacological Sciences, University of Toyama; and ${ }^{3}$ Department of Neurology, Toyama University Hospital, Toyama, Japan
\end{abstract}

\begin{abstract}
OBJECTIVE The roles of endothelial progenitor cells (EPCs) in the development of carotid plaque are still obscure. This study aimed to clarify this by assessing the histological findings of specimens obtained from carotid endarterectomy.

METHODS This study included 34 patients who underwent carotid endarterectomy. MR imaging was performed to semiquantitatively analyze the components of the carotid plaques in all patients. The surgical specimens were subjected to immunohistochemistry. The distributions of the CD34-, CD133-, VEGF-2R-positive cells in the carotid plaques were precisely analyzed, and their number was quantified. Simultaneously, the CD34-positive microvessels were localized.

RESULTS The plaque component was judged as lipid-rich plaque in 19 patients, intraplaque hemorrhage (IPH) in 11 patients, and fibrous plaque in 4 patients. The CD34-positive microvessels were densely distributed in the plaque shoulder and interface-to-media regions. The CD34-, CD133-, and VEGF-2R-positive cells were mainly localized around the CD34-positive microvessels. The number of CD34-positive microvessels significantly correlated with the number of CD34-, CD133-, and VEGF-2R-positive cells $(R=0.308, p=0.009 ; R=0.324, p=0.006$; and $R=0.296, p=0.013$, respectively). Vulnerable plaques (lipid-rich and IPH) had significantly higher numbers of the CD34-positive microvessels ( $p=0.007$ ) and CD34-, CD133-, and VEGF-2R-positive cells than fibrous plaques ( $p=0.031, p=0.013$, and $p=0.002$ ).

CONCLUSIONS These findings strongly suggest that neovascularization in the plaque shoulder and interface-to-media regions may play a key role in delivering EPCs from the peripheral blood to the carotid plaque, promoting the growth of carotid plaque. Furthermore, the invaded EPCs, especially the CD133-positive immature EPCs, may be related to plaque vulnerability.

http://thejns.org/doi/abs/10.3171/2015.10.JNS151500
\end{abstract}

KEY WORDS carotid stenosis; endothelial progenitor cells; plaque; neovascularization

$\mathrm{W}$ ORLDWIDE, carotid stenosis is one of the most frequent causes of ischemic stroke. The annual ischemic stroke risk for patients with carotid stenosis is around $2 \%$ to $5 \% .^{11,20}$ Inflammatory processes, arterial remodeling, and proliferation of smooth muscle cells are the dominant features of active atherosclerotic lesions and known to be responsible for plaque rupture and the development of ischemic stroke. ${ }^{17}$ In particular, plaque neovascularization, intraplaque hemorrhage (IPH), and plaque rupture are highly interrelated events that precipitate the evolution and clinical expression of atherothrombotic diseases. ${ }^{14,17,27,28}$ These events do not occur in hypercholesterolemic mice-the most commonly used model for atherosclerosis - and thus most relevant, albeit limited, data on IPH come from clinical studies and analyses of human tissues.

During the last decade, neovascularization in plaques has been the target of intense investigations because it is the critical source of IPH and plaque rupture and is ultimately responsible for the sudden onset of stroke..$^{14,17,27,28}$ Therefore, it is vital to identify new angiogenic factors and signaling pathways that can facilitate the development of diagnostic methods and tailored therapies for plaque stabilization.

Convincing evidence suggests that neovascularization is not solely the result of the proliferation of local endothelial cells (angiogenesis), but also involves bone marrowderived circulating endothelial progenitor cells (EPCs). ${ }^{16}$

ABBREVIATIONS EPC = endothelial progenitor cell; FOV = field of view; IPH = intraplaque hemorrhage; TOF = time of flight.

SUBMITTED June 28, 2015. ACCEPTED October 28, 2015.

INCLUDE WHEN CITING Published online February 12, 2016; DOI: 10.3171/2015.10.JNS151500. 
These cells are able to proliferate and differentiate into functionally mature endothelial cells, therefore playing an important role in the regeneration of ischemic tissue and maintenance of endothelial integrity. ${ }^{1,2}$ The number of circulating EPCs increases after ischemic stroke, revealing the importance of EPC-mediated vessel repair as a physiological response of the organism to vascular injury. ${ }^{1,2}$ EPCs are believed to repair and rejuvenate the arteries even under homeostatic physiological conditions; however, they may also contribute to lesion formation by influencing plaque stability in advanced atherosclerotic plaque under specific pathological conditions. ${ }^{4,29}$ However, little is known about the effects of EPCs on the course of atherosclerotic disease in humans. Therefore, it is fundamental to understand the regulatory networks that control endothelial progenitor differentiation, as both basic and translational research, in order to develop innovative therapeutic avenues for atherosclerotic disease. Based on these observations, this study aimed to assess the hypothesis that EPCs may also play a crucial role in the development of carotid plaques and trigger the onset of ischemic stroke, in addition to their physiological functions. In this study, the expression of EPC-specific cell surface markers, including CD34, VEGFR-2, and CD133, was confirmed by immunohistology. ${ }^{2,10,22}$

\section{Methods \\ Patients}

The Institutional Review Board at the Toyama University Hospital approved this study using human carotid plaque samples.

This study included a total of 34 patients who underwent carotid endarterectomy at our hospital between July 2012 and July 2014. There were 30 male and 4 female patients. The mean patient age was $73.1 \pm 6.7$ years. Of these, 4 patients were asymptomatic, and the other 30 patients developed transient ischemic attack or ischemic stroke. The mean degree of stenosis was $76.2 \% \pm 19.5 \%$ (range $52 \%-99 \%$ ). The surgical indications were based on the International Guidelines for Symptomatic and Asymptomatic Carotid Stenosis. ${ }^{3,8}$ Patient data were obtained via standardized questionnaires and preoperative admission charts. The degree of stenosis was evaluated using 3D CT angiography (GCA9300, Toshiba) and expressed as a percentage according to the North American Symptomatic Carotid Surgery Trial (NASCET) methods..$^{21}$ In this study, stenosis that was greater than or equal to $70 \%$ was defined as high-grade stenosis and less than $70 \%$ was defined as low-grade stenosis. In this study, 2 certified neurosurgeons (D.K. and S.K.), who were blinded to all clinical, pathological, and imaging findings, evaluated the clinical data of all patients independently and resolved disagreements by consensus.

\section{Immunohistological Analysis}

The specimens were fixed in $4 \%$ formaldehyde, embedded in paraffin, and 4- $\mu$ m-thick axial slices were prepared. The section with the largest plaque burden was classified as the culprit lesion and subjected to subsequent staining. The deparaffinized sections were processed for antigen re- trieval for 2 minutes in a pressure pot. Immunohistochemical analysis was used to identify the neovascularized microvessels and EPCs in the carotid plaques. In this study, the expression levels of EPC-specific cell-surface markers, including CD34, VEGFR-2, and CD133, were confirmed by immunohistology. ${ }^{2,10,22}$ Briefly, each section was treated with the primary antibody against CD34 (mouse monoclonal, dilution 1:100; BD Bioscience Pharmingen), VEGFR-2 (rabbit monoclonal, dilution 1:100; Cell Signaling Technology, Inc.), or CD133 (rat monoclonal, dilution 1:35; Abcam) for 40 minutes at $24^{\circ} \mathrm{C}$ and then the Envision polymer of DAKO EnVision+Kit (DAKO Cytomation) for 60 minutes. The DAB Chromogen of the DAB Substitute Kit (DAKO Cytomation) was applied for 3 to 4 minutes, and hematoxylin was used for counterstaining.

Double-fluorescence immunohistochemistry was also used to characterize the EPCs in carotid plaques. Briefly, the sections were treated with monoclonal antibodies against CD34 (mouse monoclonal, dilution 1:100; BD Bioscience Pharmingen) and VEGFR-2 (rabbit monoclonal, dilution 1:100; Cell Signaling Technology, Inc.) or against CD34 and CD133 (rat monoclonal, dilution 1:35; Abcam) at $25^{\circ} \mathrm{C}$ for 1 hour. Goat anti-mouse IgG $\mathrm{H} \& \mathrm{~L}$ secondary antibody (Alexa Fluor 555 conjugate) and donkey polyclonal secondary antibody to rabbit $\operatorname{IgG} \mathrm{H} \& \mathrm{~L}$ (Alexa Fluor 488) were added to identify their immunoreactivities. The fluorescence emitted was observed through each appropriate filter on fluorescence microscopy and digitally photographed using a cooled charge-coupled device camera equipped to the microscope (model BZ-9000, Keyence Co.).

In the immunohistological analysis, CD34-positive tube-like formation was defined as a microvessel, as reported before. ${ }^{30,32}$

The carotid plaque was divided into 4 regions (shoulder, bottom, core, and interface-to-media region), as described previously. ${ }^{12}$ The 4 regions are represented in Fig. 1. The CD34-positive microvessels and the CD34-, CD133-, and VEGF-2R-positive cells were counted in each region using the cell counter tool of ImageJ software (National Institutes of Health). In this study, the positive cell count was performed by a certified neurosurgeon (D.K.).

\section{MR Imaging of Carotid Plaques}

In all patients, the plaque components were precisely evaluated using a 1.5-T MR apparatus, as described previously. ${ }^{13}$ For plaque characterization, long-axis and axial images of the carotid artery were obtained from the 3D gradient-echo sequence and the area with the highest stenotic degree. 3D time-of-flight (TOF) MR angiography was acquired through dual carotid bifurcations in the axial plane. The imaging sequences were as follows. For 3D TOF, the field of view (FOV) was $220 \mathrm{~mm} / 87.5 \%$, TR was $23 \mathrm{msec}$, and TE was $7 \mathrm{msec}$. For the T1-weighted images, FOV was $200 \mathrm{~mm} / 100 \%$, TR was $500 \mathrm{msec}$, and TE was $11 \mathrm{msec}$. The slice thickness was $1.2 \mathrm{~mm}$ for 3D TOF and $4 \mathrm{~mm}$ for the T1-weighted images. The plaque component was defined as highly intense when the plaque displayed a signal intensity $>200 \%$ of muscle intensity at any section in the plaque; otherwise, the plaque was categorized as isointense. The plaque was considered fibrous when it 


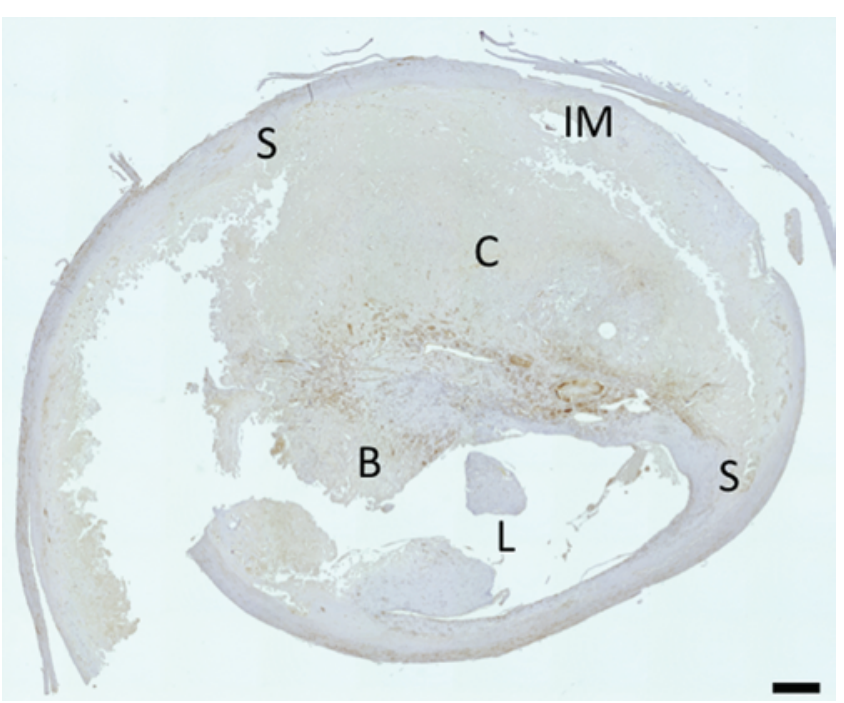

FIG. 1. Photomicrograph of immunohistochemistry against CD34. The plaque is divided into 4 regions; shoulder (S), bottom (B), core (C), and interface-to-media (IM). L = lumen. Scale bar $=500 \mu \mathrm{m}$. Figure is available in color online only.

was isointense on both T1-weighted and TOF images. The plaque was considered lipid-rich when they had high signal intensity on T1-weighted images but were isointense on TOF images. The plaque was considered to show IPH when the intensity was high on both T1-weighted and TOF images. ${ }^{23,26,31}$ In the present study, plaques with IPH and lipid-rich plaque were defined as vulnerable plaques, whereas fibrous plaques were defined as stable plaques.

\section{Statistical Analysis}

Continuous data were statistically analyzed, using 1-factor ANOVA followed by post hoc Tukey-Kramer test, Pearson correlation coefficient, or Mann-Whitney U-test, as appropriate. Values of $\mathrm{p}<0.05$ were considered statistically significant.

\section{Results}

\section{Distribution of CD34-Positive Microvessels}

Immunohistochemistry revealed that the CD34-positive microvessels were distributed within the plaque, although their density widely varied among patients. They were not contiguous with the lumina, strongly suggesting that a majority of them originated from the adventitial vasa vasorum (Fig. 2).

The total number of CD34-positive microvessels widely varied among patients, ranging from 12 to 381 with a mean value of $181.2 \pm 81.9$. In addition, their local distribution also differed among the 4 regions. Their numbers were $2.1 \pm 1.1$ in necrotic core, $102 \pm 61.3$ in shoulder, 20.0 \pm 21.7 in bottom, and $77.1 \pm 31.7$ in the interface-to-media region. The number of CD34-positive microvessels was significantly larger in the shoulder than in the core and bottom ( $\mathrm{p}=0.002$ and $\mathrm{p}=0.005$, respectively). Moreover, the numbers of CD34-positive microvessels were also significantly larger in the interface-to-media than in the core and bottom ( $p=0.003$ and $p=0.008$, respectively). Thus, plaque neovascularization was more distinct in the shoulder and interface-to-media region.

\section{Distribution of EPCs}

Immunohistochemistry revealed that the CD34-positive cells were densely localized around the CD34-positive microvessels in the carotid plaques (Fig. 2A). Likewise, CD133- and VEGFR-2-positive cells were also distributed in the same manner (Fig. 2B and C). Furthermore, double-fluorescence immunohistochemistry demonstrated that the CD34-positive cells were also positive for either VEGFR-2 or CD133, suggesting that they are EPCs (Fig. 3).

The total number of CD34-, CD133-, and VEGF-2Rpositive cells varied widely among patients. The number of CD34-positive cells ranged from 88 to 501 (mean 349.5 \pm 144.8 ), that of CD133-positive cells ranged from 12 to 228 mean $(118.2 \pm 42.1)$, and that of VEGFR-2-positive cells ranged from 79 to 471 (mean $312.1 \pm 122.4$ ).

As shown in Fig. 4, the local distributions of the CD34-, CD133-, and VEGFR-2-positive cells also differed among the 4 regions. The number of the CD34positive cells was $4.2 \pm 5.1$ in the core, $221.1 \pm 98.1$ in shoulder, $44.8 \pm 31.3$ in bottom, and $131.4 \pm 73.3$ in interface-to-media region. Thus, the number of CD34-positive cells was significantly larger in the shoulder than in core and bottom $(p=0.001$ and $p=0.005$, respectively). Likewise, the number of CD34-positive cells was significantly larger in the interface-to-media region than in the core and bottom ( $p=0.002$ and $p=0.004$, respectively). Furthermore, the number of VEGFR-2-positive cells was 1.8 \pm 2.1 in the necrotic core, $211.4 \pm 130.4$ in the shoulder, $28.9 \pm 11.4$ in the bottom, and $108.2 \pm 81.2$ in the interface-to-media region. The number of VEGF-2R-positive cells was significantly larger in the shoulder than in the core and bottom ( $p=0.002$ and $p=0.005$, respectively). The number of VEGF-2R-positive cells was significantly larger in the interface-to-media region than in the core and bottom ( $p=0.003$ and $p=0.007$, respectively). The number of CD133-positive cells was $0.6 \pm 1.8$ in the core, $66.8 \pm 30.3$ in the shoulder, $8.1 \pm 11.7$ in the bottom, and $40.3 \pm 33.1$ in the interface-to-media region. The number of CD133-positive cells was significantly larger in the shoulder than in the core and bottom $(\mathrm{p}=0.001$ and $\mathrm{p}$ $=0.004$, respectively). Moreover, the number of CD133positive cells was significantly larger in the interface-tomedia region than in the core and bottom $(p=0.003$ and $\mathrm{p}=0.008$, respectively). Thus, the EPCs were primarily distributed in the shoulder and interface-to-media regions of carotid plaques.

\section{Relationship Between Microvessels and EPCs}

As shown in Fig. 5, there were significant correlations between the number of the CD34-positive microvessels and CD34-, CD133-, and VEGF-2R-positive cells in the carotid plaques $(\mathrm{R}=0.308, \mathrm{p}=0.009 ; \mathrm{R}=0.324, \mathrm{p}=$ 0.006 ; and $R=0.296, p=0.013$, respectively). Thus, the EPCs were densely identified in neovascularization-rich carotid plaques. 

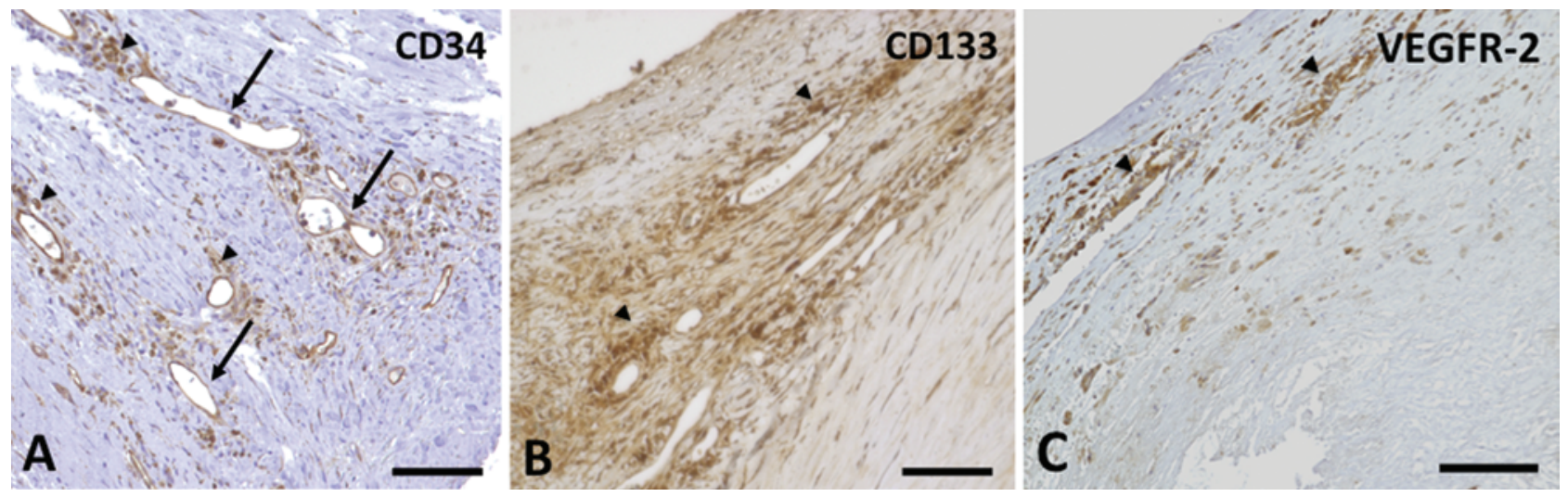

FIG. 2. Photomicrographs of immunohistochemistry against CD34, CD133, and VEGFR-2. A: CD34-positive microvessels are densely distributed within the carotid plaque (arrows). Note that the CD34-positive cells are distributed around the CD34-positive microvessels (arrowheads). Both CD133-positive (B) and VEGFR-2-positive cells (C) are also distributed around the CD34-positive microvessels (arrowheads). Scale bar $=100 \mu \mathrm{m}$. Figure is available in color online only.

\section{Plaque Vulnerability}

Preoperative MR imaging revealed lipid-rich plaques in 19 patients, IPH in 11 patients, and fibrous plaque in 4 patients. Thus, 30 plaques were categorized as vulnerable plaques and the remaining 4 as stable plaques. Of these, 3 stable plaques $(75.0 \%)$ and 1 vulnerable plaque $(3.3 \%)$ were asymptomatic.

Statistical analysis showed that the vulnerable plaques had a significantly larger number of CD34-positive microvessels than stable plaques: $320.9 \pm 122.9$ and 120.3 \pm 91.1 , respectively ( $\mathrm{p}=0.007$; Fig. 6). The numbers of CD34-, VEGFR-2-, and CD133-positive cells were also significantly larger in vulnerable plaques than in stable plaques ( $\mathrm{p}=0.031$ for CD34, $\mathrm{p}=0.013$ for VEGFR-2, and $\mathrm{p}=0.002$ for CD133). Thus, the number of EPCs corre- lated very well with plaque vulnerability in the carotid artery. Of these, CD133 was the most reliable predictor for plaque vulnerability.

On the basis of the assessment of symptomatology, there were significant differences in the numbers of CD34-, VEGFR-2-, and CD133-positive cells and CD34positive microvessels between high symptomatic stenosis and asymptomatic stenosis $(\mathrm{p}=0.021$ for CD34-positive cells, $p=0.011$ for VEGFR-2-positive cells, $p=0.003$ for CD133-positive cells, and $\mathrm{p}=0.019$ for CD34-positive microvessels).

On the basis of the assessment of stenosis degree, there were no significant differences in the numbers of CD34-, VEGFR-2-, and CD133-positive cells and CD34-positive microvessels between high-grade stenosis and low-grade
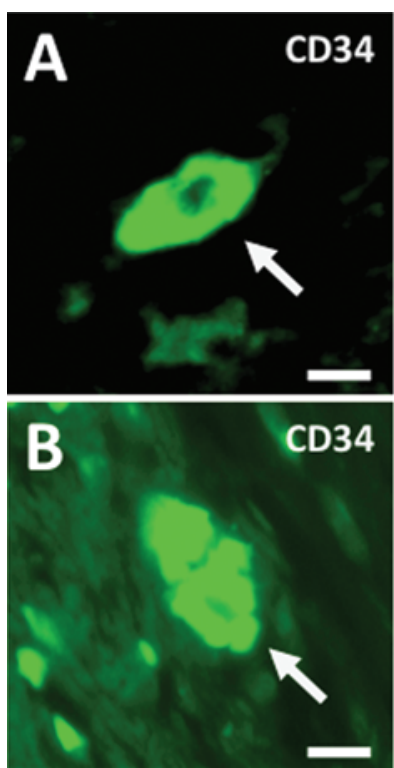
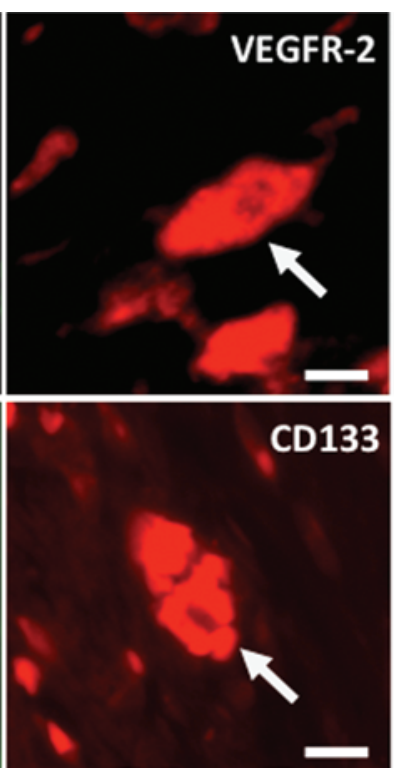
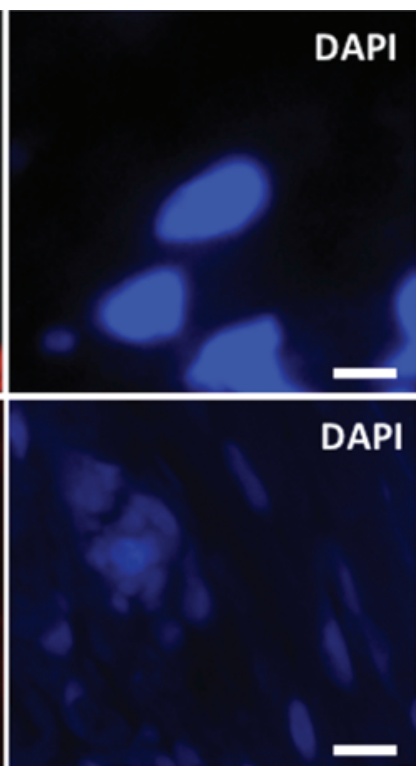

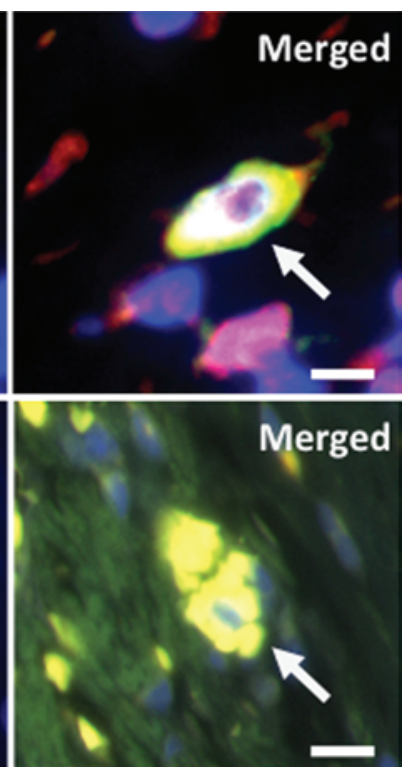

FIG. 3. Photomicrographs of double-fluorescence immunohistochemistry against CD34, VEGFR-2, and DAPI (A) and against CD34, CD133, and DAPI (B). Note that the CD34-positive cells are also positive for VEGFR-2 and CD133. Scale bar $=20 \mu \mathrm{m}$. Figure is available in color online only. 
stenosis $(\mathrm{p}=0.099$ for CD34-positive cells, $\mathrm{p}=0.155$ for VEGFR-2-positive cells, $\mathrm{p}=0.103$ for CD133-positive cells, and $\mathrm{p}=0.211$ for CD34-positive microvessels).

\section{Discussion}

The principal findings reported here are, first, that EPCs are densely distributed around the microvessels in carotid plaques. Second, both the microvessels and surrounding EPCs are unevenly localized in the shoulder and interface-to-media regions. Microvessels are not contiguous with the arterial lumen and, therefore, are considered primarily derived from the vasa vasorum during the development of carotid plaques. In addition, the homing pathway of the microvessels under pathological conditions appears completely different from that under healthy physiological conditions. Third, vulnerable plaques contain more EPCs and microvessels than stable or fibrous plaques. Finally, CD133-positive cells may be the most reliable predictor of vulnerable plaques in the carotid artery.

\section{The Roles of EPCs and Microvessels in Carotid Plaque}

EPCs are known to repair and rejuvenate the arteries under physiological conditions; however, under specific pathological conditions, they may also contribute to lesion formation by influencing plaque stability in advanced atherosclerosis. ${ }^{6,19}$ Recently, EPCs have been described as a subpopulation of pluripotent cells within the peripheral blood from the bone marrow, capable of differentiating into endothelial cells. ${ }^{2}$ Under physiological conditions, the integrity of the endothelial monolayer can be maintained by the replication of adjacent cells. In particular, progenitor cell homing prevented damage to the endothelium's integrity in aging-induced oxidative stress. Under pathological conditions such as atherosclerosis and cancer, however, EPCs are known to play an important role in disease progression. In fact, the present study demonstrated that EPCs coexisting with microvessels were mainly observed in the shoulder and interface-to-media regions. Moreover, the number of EPCs identified in the carotid plaque strongly depends on the quantity of the microvessels, suggesting that EPCs might be supplied via the newly developed microvessels from the vasa vasorum. The vasa vasorum acts not only as the blood conduit tube but also as a major EPC reservoir in advanced plaques. Since the EPCs play an important role in neovascularization, increasing quantities of these cells may promote neovascularization in the plaque and negatively impact plaque stability. These negative interactions may result in plaque growth and/or IPH and provoke ischemic stroke. Kumamoto et al. reported that adventitia-derived intimal vessels occurred approximately 28 times more frequently than lumen-derived vessels in coronary plaques..$^{14}$ These data agree with the present findings that adventitia-derived angiogenesis in conjunction with the EPCs' ability to home to plaques via the microvessels from the vasa vasorum may be a key mechanism in the development of carotid plaques. Under normal conditions, the vasa vasorum forms a network of microvasculature on the surfaces of large arteries and supplies oxygen and nutrients to the outer layers of the arterial wall. Under pathological conditions, however,

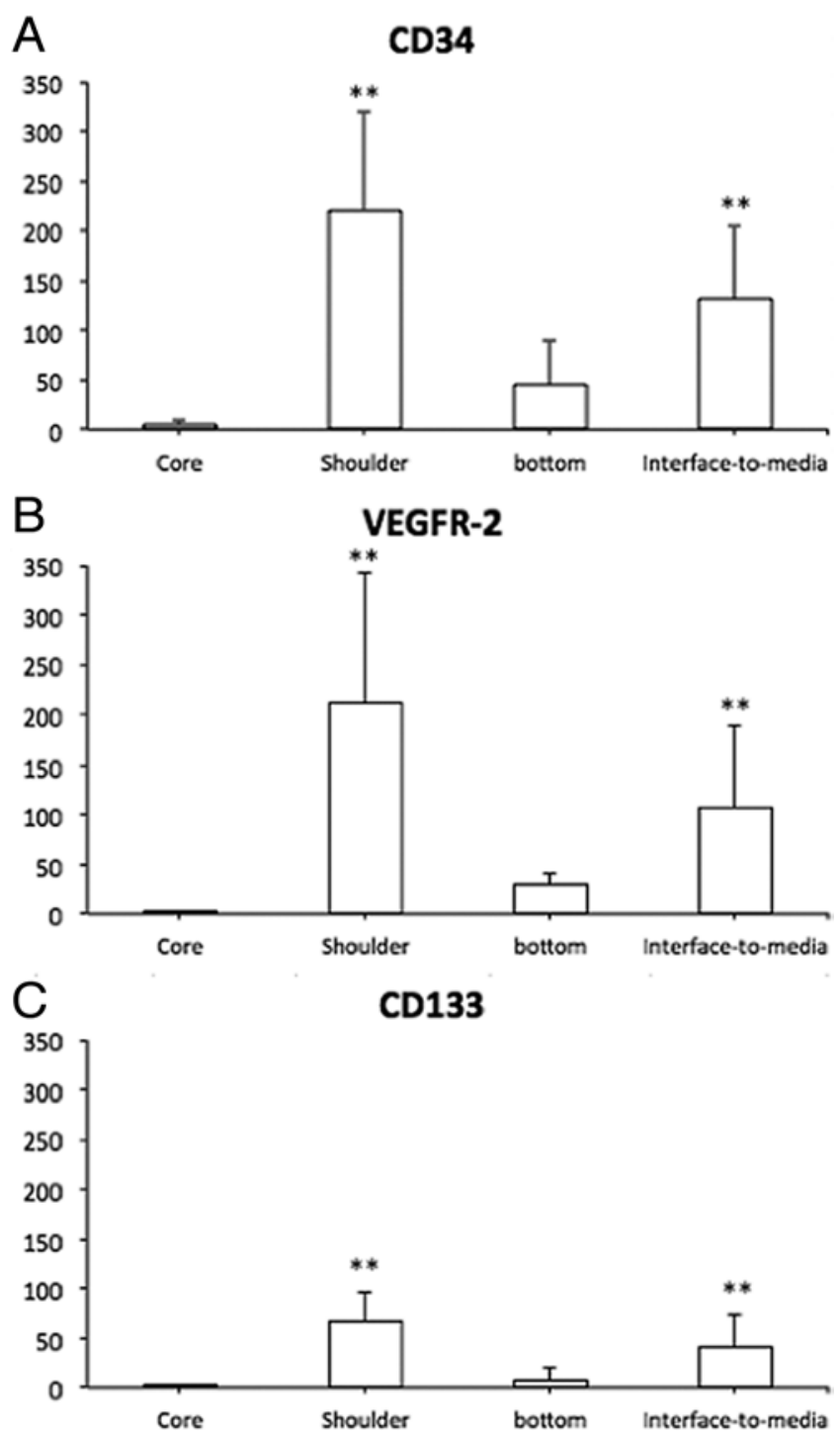

FIG. 4. Bar graphs show the number of CD34-positive, VEGFR-2positive, and CD133-positive cells in the core, shoulder, bottom, and interface-to-media region of the carotid plaque. ${ }^{* *} p<0.01$ in comparison with the core and bottom (1-factor ANOVA followed by the Tukey-Kramer test). The whiskers indicate the standard deviation.

the role of EPCs is dramatically changed. Therefore, the vasa vasorum may be one of the major therapeutic targets to preventing the growth of carotid plaque. According to Moulton et al., it may be valuable to block angiogenesis via the vasa vasorum with angiostatins in order to reduce the accumulation of macrophages in plaques and prevent the progression of atherosclerosis. ${ }^{18}$ Taken together, EPCderived angiogenesis may play a key role in plaque growth and instability by promoting the accumulation of both EPCs and macrophages.

In the present study, the majority of the EPCs and microvessels were found in the shoulder and interface-tomedia regions. The data strongly suggest that the shoulder and interface-to-media regions are the responsible sites for importing EPCs from the peripheral blood and advancing 

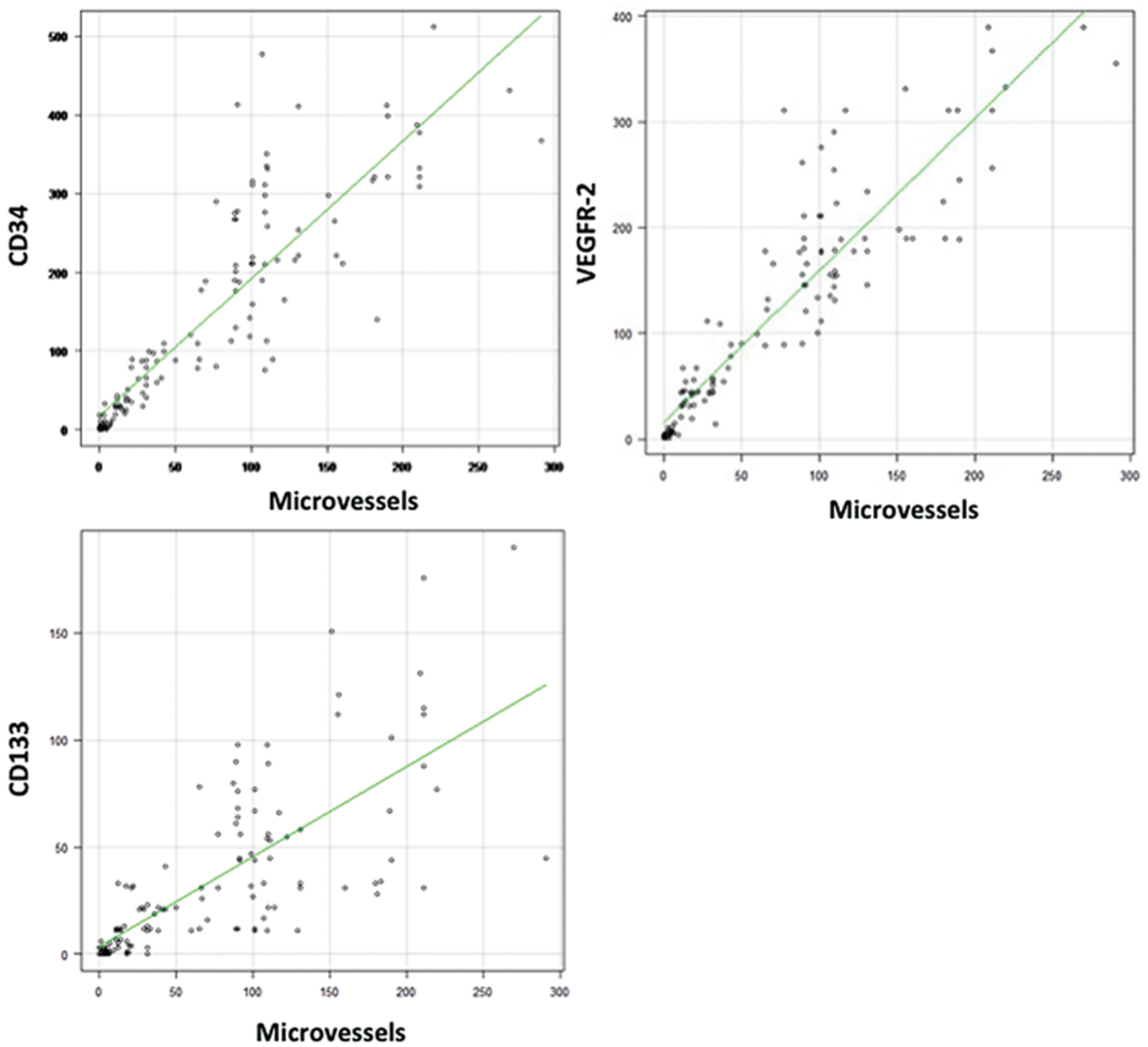

FIG. 5. The scatter plots demonstrate the significant correlations between CD34-positive microvessels and CD34-positive, VEGFR-2-positive, and CD133-positive cells $(R=0.308, p=0.009 ; R=0.324, p=0.006 ; R=0.296, p=0.013$, respectively). Figure is available in color online only.

plaque growth. These regions, may be novel therapeutic and diagnostic targets for carotid plaque treatment, although further studies are warranted prior to clinical application.

\section{Plaque Components and EPCs Levels}

In the current study, vulnerable plaques had a significantly larger number of CD34-, VEGFR-2-, and CD133positive cells, as well as the CD34-positive microvessels, than stable plaques. This finding suggests that the increase in EPCs in carotid plaques was closely associated with plaque vulnerability. Angiogenesis is a pivotal defense mechanism for counteracting hypoxia and necessary for plaque regression. However, the neovessels in the plaque are also known to be susceptible to IPH. In this scenario, EPCs may play an important role in the development of plaque instability. Using flow cytometry, some investigators have analyzed the circulating EPCs in the peripheral blood and reached the conclusion that the circulating EPC level is reduced in patients with advanced carotid atherosclerosis. ${ }^{15,25}$ Therefore, the circulating EPCs may accumulate in carotid plaques, contribute to neovascularization, and decrease stability. Finally, the circulating EPC level may be decreased in patients at higher risk for ischemic stroke.

\section{Biological Properties of EPCs in Carotid Plaque}

In the present study, there were strongly significant differences in the number of CD133-positive cells between stable and vulnerable plaques. Asahara et al. ${ }^{2}$ first reported that EPCs are considered to express CD34 and VEGFR-2. The subsequent discovery of another marker, CD133, allowed further categorization of the EPC population into 2 subpopulations: CD133-positive/CD34-positive/ VEGFR-2-positive cells and CD133-negative/CD34-pos- 


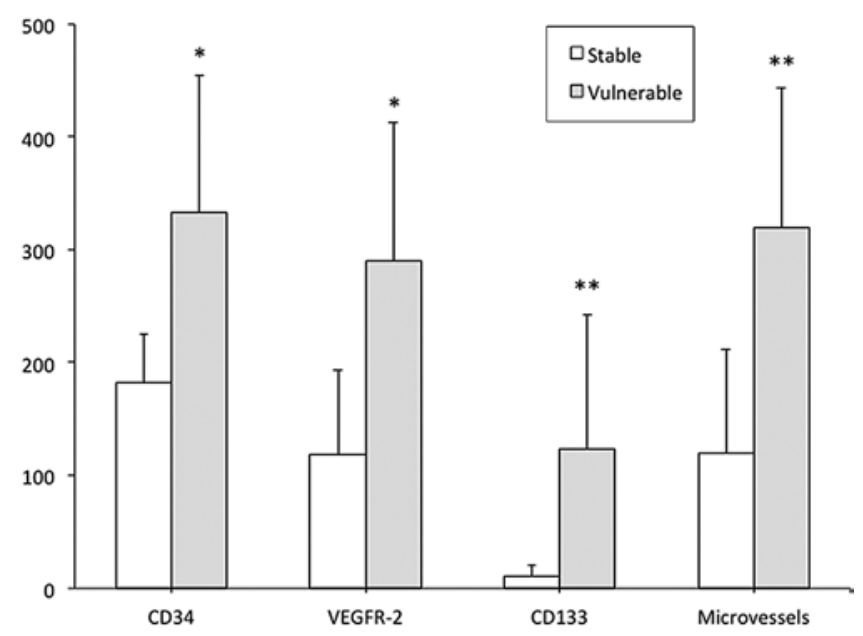

FIG. 6. Bar graph shows the number of CD34-positive, VEGFR-2-positive, and CD133-positive cells and CD34-positive microvessels in stable and vulnerable carotid plaques. ${ }^{*} p<0.05,{ }^{* *} p<0.01$ between 2 groups. The whiskers indicate the standard deviation.

itive/VEGFR-2-positive cells. The former presumably consists of more primordial progenitors, and the latter is potentially more mature and further differentiated along the endothelial lineage.,22 Thus, EPCs are now thought of as a heterogeneous population of progenitors., ${ }^{7,222,24}$ The present data strongly demonstrate that vulnerable plaques include more immature EPCs and CD133-positive/CD34positive/VEGFR-2-positive cells, suggesting that their homing may be related to plaque instability. Therefore, immature EPCs can be a therapeutic target for unstable plaques at higher risk for the onset of ischemic stroke. As the EPCs mature toward endothelial cells, they gradually lose CD133 expression but they upregulate the expression of adhesion molecules and acquire endothelial functions such as low-density lipoprotein uptake and nitric oxide synthesis. In the present study, both CD133-negative/ CD34-positive/VEGFR-2-positive cells and CD133-positive/CD34-positive/VEGFR-2-positive cells coexisted in unstable plaques with high vascular density, whereas only CD133-negative/CD34-positive/VEGFR-2-positive cells existed in stable plaque with low vascular density. Thus, premature CD133-expressing EPCs are most likely mobilized to unstable plaques from the peripheral blood via the neovessels in plaques. Recently, Herrmann et al. suggested that CD34-positive/CD133-positive EPCs are enriched in the bone marrow, promote neovascularization, and have high angiogenic potential. ${ }^{10} \mathrm{Chi}$ et al. reported that the CD34-positive/CD133-positive cells may predict the development and severity of atherosclerosis at the cellular level. ${ }^{5}$ Taken together, these individual reports may explain the positive relationship between the number of CD133-positive EPCs and the number of microvessels in carotid plaques.

As limitations, despite the limited number of samples, this study accurately reflects and encapsulates the immunohistological characteristics of carotid plaques. Future studies should encompass larger populations and focus on the therapeutic control of EPCs in these patients.

\section{Conclusions}

Using carotid plaque specimens, the findings provide strong evidence that EPCs travel preferentially to vasa vasorum-derived microvessels and promote angiogenesis. Despite the limited sample size, this study accurately reflects and encapsulates the histological characteristics of carotid plaques. These findings strongly suggest that neovascularization in the plaque shoulder and interfaceto-media regions may play a key role in delivering EPCs from the peripheral blood to the carotid plaque, promoting the growth of carotid plaques. Furthermore, the invaded EPCs, especially CD133-positive immature EPCs, may be related to plaque vulnerability. In light of the observed higher expression of EPCs in plaques from patients with symptomatic carotid stenosis, it is logical to speculate that EPCs may also contribute to the onset of ischemic stroke. Future studies should encompass larger populations and focus on therapeutic control of EPCs in these patients.

\section{References}

1. Asahara T, Masuda H, Takahashi T, Kalka C, Pastore C, Silver M, et al: Bone marrow origin of endothelial progenitor cells responsible for postnatal vasculogenesis in physiological and pathological neovascularization. Circ Res 85:221-228, 1999

2. Asahara T, Murohara T, Sullivan A, Silver M, van der Zee R, $\mathrm{Li} \mathrm{T}$, et al: Isolation of putative progenitor endothelial cells for angiogenesis. Science 275:964-967, 1997

3. Barnett HJ, Taylor DW, Eliasziw M, Fox AJ, Ferguson GG, Haynes RB, et al: Benefit of carotid endarterectomy in patients with symptomatic moderate or severe stenosis. North American Symptomatic Carotid Endarterectomy Trial Collaborators. N Engl J Med 339:1415-1425, 1998

4. Briasoulis A, Tousoulis D, Antoniades C, Papageorgiou N, Stefanadis C: The role of endothelial progenitor cells in vascular repair after arterial injury and atherosclerotic plaque development. Cardiovasc Ther 29:125-139, 2011

5. Chi J, Hong X, Wang Y, Zhao J, Yang W: Inverse correlation between circulating endothelial progenitor cells with CD34+CD133+ and the severity of coronary atherosclerosis assessed by Syntax score. Am J Med Sci 347:457-462, 2014

6. Eguchi M, Masuda H, Asahara T: Endothelial progenitor cells for postnatal vasculogenesis. Clin Exp Nephrol 11:1825,2007

7. Gehling UM, Ergün S, Schumacher U, Wagener C, Pantel K, Otte M, et al: In vitro differentiation of endothelial cells from AC133-positive progenitor cells. Blood 95:3106-3112, 2000

8. Halliday A, Mansfield A, Marro J, Peto C, Peto R, Potter $\mathrm{J}$, et al: Prevention of disabling and fatal strokes by successful carotid endarterectomy in patients without recent neurological symptoms: randomised controlled trial. Lancet 363:1491-1502, 2004

9. Handgretinger R, Gordon PR, Leimig T, Chen X, Buhring HJ, Niethammer D, et al: Biology and plasticity of CD133+ hematopoietic stem cells. Ann N Y Acad Sci 996:141-151, 2003

10. Herrmann M, Binder A, Menzel U, Zeiter S, Alini M, Verrier S: CD34/CD133 enriched bone marrow progenitor cells promote neovascularization of tissue engineered constructs in vivo. Stem Cell Res (Amst) 13 (3 Pt A):465-477, 2014

11. Inzitari D, Eliasziw M, Gates P, Sharpe BL, Chan RK, Meldrum HE, et al: The causes and risk of stroke in patients with asymptomatic internal-carotid-artery stenosis. North American Symptomatic Carotid Endarterectomy Trial Collaborators. N Engl J Med 342:1693-1700, 2000 
12. Ionita MG, van den Borne P, Catanzariti LM, Moll FL, de Vries JP, Pasterkamp G, et al: High neutrophil numbers in human carotid atherosclerotic plaques are associated with characteristics of rupture-prone lesions. Arterioscler Thromb Vasc Biol 30:1842-1848, 2010

13. Kashiwazaki D, Akioka N, Kuwayama N, Noguchi K, Tanaka K, Kuroda S: Pathophysiology of acute cerebrovascular syndrome in patients with carotid artery stenosis: a magnetic resonance imaging/single-photon emission computed tomography study. Neurosurgery 76:427-434, 2015

14. Kumamoto M, Nakashima Y, Sueishi K: Intimal neovascularization in human coronary atherosclerosis: its origin and pathophysiological significance. Hum Pathol 26:450-456, 1995

15. Lau KK, Chan YH, Yiu KH, Li SW, Tam S, Lau CP, et al: Burden of carotid atherosclerosis in patients with stroke: relationships with circulating endothelial progenitor cells and hypertension. J Hum Hypertens 21:445-451, 2007

16. Moon JH, Chae MK, Kim KJ, Kim HM, Cha BS, Lee HC, et al: Decreased endothelial progenitor cells and increased serum glycated albumin are independently correlated with plaque-forming carotid artery atherosclerosis in type 2 diabetes patients without documented ischemic disease. Circ J 76:2273-2279, 2012

17. Moreno PR, Purushothaman KR, Sirol M, Levy AP, Fuster $\mathrm{V}$ : Neovascularization in human atherosclerosis. Circulation 113:2245-2252, 2006

18. Moulton KS, Vakili K, Zurakowski D, Soliman M, Butterfield C, Sylvin E, et al: Inhibition of plaque neovascularization reduces macrophage accumulation and progression of advanced atherosclerosis. Proc Natl Acad Sci U S A 100:4736-4741, 2003

19. Murasawa S, Asahara T: Endothelial progenitor cells for vasculogenesis. Physiology (Bethesda) 20:36-42, 2005

20. Norris JW, Zhu CZ, Bornstein NM, Chambers BR: Vascular risks of asymptomatic carotid stenosis. Stroke 22:1485-1490, 1991

21. North American Symptomatic Carotid Endarterectomy Trial Collaborators: Beneficial effect of carotid endarterectomy in symptomatic patients with high-grade carotid stenosis. $\mathbf{N}$ Engl J Med 325:445-453, 1991

22. Peichev M, Naiyer AJ, Pereira D, Zhu Z, Lane WJ, Williams M, et al: Expression of VEGFR-2 and AC133 by circulating human $\mathrm{CD} 34^{+}$cells identifies a population of functional endothelial precursors. Blood 95:952-958, 2000

23. Saito A, Sasaki M, Ogasawara K, Kobayashi M, Hitomi J, Narumi S, et al: Carotid plaque signal differences among four kinds of T1-weighted magnetic resonance imaging techniques: a histopathological correlation study. Neuroradiology 54:1187-1194, 2012

24. Shmelkov SV, St Clair R, Lyden D, Rafii S: AC133/CD133/ Prominin-1. Int J Biochem Cell Biol 37:715-719, 2005

25. Stein A, Montens HP, Steppich B, Busch G, Brandl R, Ott I: Circulating endothelial progenitor cells decrease in patients after endarterectomy. J Vasc Surg 48:1217-1222, 2008

26. Takaya N, Yuan C, Chu B, Saam T, Polissar NL, Jarvik GP, et al: Presence of intraplaque hemorrhage stimulates progression of carotid atherosclerotic plaques: a high-resolution magnetic resonance imaging study. Circulation 111:27682775,2005

27. Truijman MT, de Rotte AA, Aaslid R, van Dijk AC, Steinbuch J, Liem MI, et al: Intraplaque hemorrhage, fibrous cap status, and microembolic signals in symptomatic patients with mild to moderate carotid artery stenosis: the Plaque at RISK study. Stroke 45:3423-3426, 2014

28. van den Oord SC, Akkus Z, Renaud G, Bosch JG, van der Steen AF, Sijbrands EJ, et al: Assessment of carotid atherosclerosis, intraplaque neovascularization, and plaque ulceration using quantitative contrast-enhanced ultrasound in asymptomatic patients with diabetes mellitus. Eur Heart J Cardiovasc Imaging 15:1213-1218, 2014

29. Vasa M, Fichtlscherer S, Adler K, Aicher A, Martin H, Zeiher AM, et al: Increase in circulating endothelial progenitor cells by statin therapy in patients with stable coronary artery disease. Circulation 103:2885-2890, 2001

30. Vavuranakis M, Sigala F, Vrachatis DA, Papaioannou TG, Filis K, Kavantzas N, et al: Quantitative analysis of carotid plaque vasa vasorum by CEUS and correlation with histology after endarterectomy. Vasa 42:184-195, 2013

31. Yoshida K, Narumi O, Chin M, Inoue K, Tabuchi T, Oda K, et al: Characterization of carotid atherosclerosis and detection of soft plaque with use of black-blood MR imaging. AJNR Am J Neuroradiol 29:868-874, 2008

32. You X, Huang P, Zhang C, Wang M, Zhang Y, Hong Y, et al: Relationship between enhanced intensity of contrast enhanced ultrasound and microvessel density of aortic atherosclerotic plaque in rabbit model. PLoS One 9:e92445, 2014

\section{Disclosures}

The authors report no conflict of interest concerning the materials or methods used in this study or the findings specified in this paper.

\section{Author Contributions}

Conception and design: Kashiwazaki. Acquisition of data: Kashiwazaki, Noguchi. Analysis and interpretation of data: Kashiwazaki, Hayashi, Noguchi, Tanaka. Drafting the article: Kashiwazaki. Critically revising the article: Kuwayama, Hayashi, Tanaka. Reviewed submitted version of manuscript: Akioka, Kuwayama, Tanaka, Kuroda. Approved the final version of the manuscript on behalf of all authors: Kashiwazaki. Statistical analysis: Kashiwazaki, Akioka, Kuwayama, Hayashi. Administrative/technical/material support: Kuwayama, Kuroda. Study supervision: Kuroda.

\section{Correspondence}

Daina Kashiwazaki, Department of Neurosurgery, Graduate School of Medicine and Pharmaceutical Science, University of Toyama, 2630 Sugitani, Toyama 930-0194, Japan. email: gktqx702@yahoo.co.jp. 